\title{
Preoperative tumor size is associated with deep myometrial invasion and lymph node metastases and is a negative prognostic indicator for patients with endometrial carcinoma
}

\author{
Kohei Nakamura ${ }^{1}$, Kentaro Nakayama ${ }^{1}$, Noriyoshi Ishikawa ${ }^{2}$, Toshiko Minamoto ${ }^{1}$, \\ Tomoka Ishibashi ${ }^{1}$, Kaori Ohnishi ${ }^{1}$, Hitomi Yamashita ${ }^{1}$, Ruriko Ono ${ }^{1}$, Hiroki \\ Sasamori ${ }^{1}$, Sultana Razia ${ }^{1}$, Mohammad Mahmud Hossain ${ }^{1}$, Shanta Kamrunnahar ${ }^{1}$, \\ Masako Ishikawa ${ }^{1}$ and Satoru Kyo ${ }^{1}$ \\ ${ }^{1}$ Department of Obstetrics and Gynecology, Shimane University School of Medicine, Izumo 6938501, Japan \\ ${ }^{2}$ Department of Organ Pathology, Shimane University School of Medicine, Izumo 6938501, Japan \\ Correspondence to: Kentaro Nakayama, email: kn88@med.shimane-u.ac.jp \\ Keywords: endometrial carcinoma; tumor size; overall survival; progression-free survival; myometrial invasion \\ Received: January 31, $2018 \quad$ Accepted: April 07, $2018 \quad$ Published: May 01, 2018
}

Copyright: Nakamura et al. This is an open-access article distributed under the terms of the Creative Commons Attribution License 3.0 (CC BY 3.0), which permits unrestricted use, distribution, and reproduction in any medium, provided the original author and source are credited.

\section{ABSTRACT}

We examined the usefulness of evaluating tumor size determined using preoperative magnetic resonance imaging (MRI) for prognosis in patients with endometrial carcinoma (EC). Patients $(N=184)$ with EC who underwent surgery at Shimane University Hospital between 1997 and 2013 were enrolled. We investigated the association between the tumor size of EC assessed prior to surgery by MRI (anteroposterior [AP], transverse [TV], and craniocaudal [CC] diameters) and various clinical parameters including deep myometrial invasion and lymph node metastases. We subsequently examined the prognostic significance of tumor size in patients with EC. Survival analysis was performed using the Kaplan-Meier method, and prognostic factors were evaluated using the Cox's proportional hazards regression model.

Multivariate analysis identified increased AP diameter as an independent negative prognostic factor for overall survival $(O S)(P=0.037)$. A long AP diameter has prognostic value and the potential to be a predictive marker for surgical outcomes in patients with EC. Furthermore, AP diameter exhibited the greatest area under the curve (AUC) (0.727) for deep myometrial invasion, and CC diameter had the greatest AUC for lymph node metastases $(0.854)$. Evaluation of tumor size parameters may aid in the identification of high-risk populations, which could improve treatment selection and patient outcomes.

\section{INTRODUCTION}

Endometrial cancer (EC) is the most common gynecologic malignancy, with an annual incidence of 320,000 and a mortality rate of 76,000 deaths per year worldwide $[1,2]$. The International Federation of Gynecology and Obstetrics (FIGO) staging system is used by clinicians for prognostication and to guide surgical management. Interestingly, patients with the same disease stage may experience very different clinical courses $[1,3]$. To understand the reasons for these differences, many investigators have evaluated the influence of various tumor attributes, such as histological subtype and FIGO stage, grade, depth of myometrial invasion, and vascular invasion on prognosis [3-5]. Unfortunately, preoperative evaluations generally require invasive, costly, and timeconsuming procedures such as fractional curettage or hysteroscopic assessment [1, 6-8]. Early detection and improvements in surgical techniques and chemotherapies have contributed to better prognoses. However, precise predictions of prognosis remain a challenge despite being necessary for guiding clinical decision-making regarding optimal treatment.

Previous studies on cervical, breast, and renal cancers have demonstrated that increased tumor size is associated with a poorer prognosis [9-12]. In their 
study, Gusberg et al. [13] reported that tumor size was an indicator of prognosis in EC and used uterine size as a surrogate marker for tumor size [13]. Many studies have since reported conflicting results when examining the usefulness of tumor size as a prognostic indicator. Several studies have demonstrated that tumor size is significantly associated with lymph node metastases and survival [1416], leading them to recommend the addition of tumor size to routine assessments for identifying low-risk endometrial cancer patients [17]. Other studies, however, have challenged such a correlation $[18,19]$. Moreover, the FIGO staging system does not mention tumor size for endometrial cancer diagnosis [20]. Soliman et al. [21] outlined factors used to guide surgical decisionmaking for patients with endometrial cancer and focused on the indications for lymphadenectomy; interestingly, the authors did not consider tumor size as part of their survey [21].

The advantages of evaluating tumor size include ease of evaluation and the lack of a need for extra resources or a skilled pathologist. In contrast, intraoperative assessment of other prognostic indicators such as grade, myometrial invasion, and lymph metastases require well-trained pathologists and additional resources, which can vary depending on whether frozen or paraffin sections are required [22, 23]. Magnetic resonance imaging (MRI) is another modality that can be used to assess tumor size; however, to our knowledge, studies examining the reproducibility of tumor size measurements by MRI are lacking. Moreover, there is currently no established optimal threshold value for risk assessment based on tumor size.

Here, the primary aim was to examine the relationship between preoperative tumor size measured by MRI and staging variables including deep myometrial invasion and lymph node metastases among patients with EC. The secondary aim was to clarify the prognostic significance of tumor size in patients with EC.

\section{RESULTS}

\section{Patient and clinical features}

A total of 184 patients with EC were enrolled. Their clinical and pathological characteristics are shown in Table 1. Regarding histology, 87.5\% (161/184) of tumors were endometrioid, $8.1 \%(15 / 184)$ were serous, and $4.3 \%$ $(8 / 184)$ were clear cell. In our analysis, we divided the histology into two categories (endometrioid vs. others).

\section{Relationships between tumor size parameters and prognosis in patients with $\mathrm{EC}$ and selection of the optimal threshold value for tumor size}

We used receiver operating characteristic (ROC) analysis to define the optimal threshold value for each tumor size measurement in the prediction of progressionfree survival (PFS) and overall survival (OS). Tumor size measurements along each of 3 orthogonal planes (anteroposterior [AP], transverse [TV], and craniocaudal [CC]) were associated with PFS and OS in Kaplan-Meier analysis. The area under the curve (AUC) for the AP diameter was 0.719 for PFS, and the threshold value was $28 \mathrm{~mm}$ (Supplementary Figure 1A); the AP diameter was significantly associated with PFS $(P<0.001)$ (Figure 1A). The AUC for the AP diameter was 0.746 for OS, and the threshold value was $28 \mathrm{~mm}$ (Supplementary Figure 1B); the AP diameter was significantly associated with OS $(P=0.001)$ (Figure 1B). The AUC for the CC diameter was 0.663 for PFS, and the threshold value was 52 $\mathrm{mm}$ (Supplementary Figure 2A); the CC diameter was significantly associated with PFS $(P=0.001)$ (Figure 1C). The AUC for the CC diameter was 0.660 for OS, and the threshold value was $52 \mathrm{~mm}$ (Supplementary Figure 2B); the CC diameter was significantly associated with OS $(P=0.012)$ (Figure 1D). The AUC for the TV diameter was 0.603 for PFS, and the threshold value was 37 mm (Supplementary Figure 3A); the TV diameter was significantly associated with $\operatorname{PFS}(P=0.013)$ (Figure 1E). The AUC for the TV diameter was 0.596 for OS, and the threshold value was $37 \mathrm{~mm}$ (Supplementary Figure 3B); the TV diameter was significantly associated with OS $(P=0.018)$ (Figure 1F).

\section{Univariate and multivariate analyses of prognostic factors in patients with EC}

The association between age, clinical stage, histological type, grade, deep myometrial invasion, lymph node metastasis, venous invasion, lymphatic invasion, and the three tumor size measurements and survival were investigated using univariate analyses. For PFS, stage $(P<0.001)$, histologic type $(P=0.015)$, grade $(P=0.003)$, deep myometrial invasion $(P=$ $0.012)$, lymph node metastasis $(P<0.001)$, venous invasion $(\mathrm{P}=0.002)$, lymphatic invasion $(P<0.001)$, AP diameter $(P=0.001), \mathrm{CC}$ diameter $(P=0.002)$, and TV diameter $(P=0.017)$ were significant predictors. Multivariate analysis revealed that advanced stage (III/ IV) (hazard ratio $[\mathrm{HR}]=4.441 ; 95 \%$ confidence interval $[\mathrm{CI}]=1.463-13.476 ; P=0.008)$ was an independent negative predictor of PFS (Table 2). For OS, as shown in Table 3 , stage $(P<0.001)$, histologic type $(P=0.027)$, grade $(P=0.033)$, lymph node metastasis $(P=0.001)$, venous invasion $(\mathrm{P}=0.017)$, lymphatic invasion $(P$ $=0.005), \mathrm{CC}$ diameter $(P<0.001)$, AP diameter $(P=$ $0.012)$, and TV diameter $(P=0.018)$ were significant predictors. Multivariate analysis showed that advanced stage (stage III/IV) (HR, 5.756; 95\% CI, 1.708-19.397; $P=0.005)$ and AP diameter (HR, 5.285; 95\% CI, $1.110-25.170 ; P=0.037)$ were significant independent negative predictors of OS (Table 3 ). 
Table 1: Clinical characteristics of the patient population $(n=184)$

\begin{tabular}{|c|c|c|}
\hline Characteristic & No. of patients & $\%$ \\
\hline \multicolumn{3}{|c|}{ Age at diagnosis, y } \\
\hline$<60$ & 93 & 51 \\
\hline$\geq 60$ & 91 & 49 \\
\hline \multicolumn{3}{|l|}{ FIGO stage } \\
\hline I, II & 143 & 78 \\
\hline III, IV & 41 & 22 \\
\hline \multicolumn{3}{|l|}{ Histology } \\
\hline Endometrioid & 161 & 88 \\
\hline Other & 23 & 12 \\
\hline \multicolumn{3}{|l|}{ Grade } \\
\hline G1 & 85 & 46 \\
\hline G2, G3 & 99 & 54 \\
\hline \multicolumn{3}{|c|}{ Myometrial invasion } \\
\hline$<1 / 2$ & 119 & 65 \\
\hline$\geq 59 /$ & 65 & 35 \\
\hline \multicolumn{3}{|l|}{ Lymph metastasis } \\
\hline No & 136 & 74 \\
\hline Yes & 19 & 10 \\
\hline Not assessed & 29 & 16 \\
\hline \multicolumn{3}{|l|}{ Venous invasion } \\
\hline No & 125 & 68 \\
\hline Yes & 59 & 32 \\
\hline \multicolumn{3}{|c|}{ Lymphatic invasion } \\
\hline No & 104 & \\
\hline Yes & 80 & \\
\hline \multicolumn{3}{|l|}{ AP diameter, mm } \\
\hline$<52$ & 146 & \\
\hline$\geq 46$ & 38 & \\
\hline \multicolumn{3}{|l|}{ TV diameter, mm } \\
\hline$<37$ & 119 & \\
\hline$\geq 19$ & 65 & \\
\hline \multicolumn{3}{|l|}{$\mathrm{CC}$ diameter, $\mathrm{mm}$} \\
\hline$<28$ & 111 & \\
\hline$\geq 11$ & 73 & \\
\hline
\end{tabular}

FIGO, International Federation of Gynecology and Obstetrics; AP, anteroposterior; TV, transverse; CC, craniocaudal. 


\section{Correlation between the three tumor size measurements and deep myometrial invasion in patients with EC}

Using the above threshold values for each tumor size measurement, ROC curves indicating the effectiveness of the diameter measurements for predicting deep myometrial invasion (Figure 2A) and lymph node metastases (Figure 2B) demonstrated that the AP diameter had the greatest AUC (0.727) for deep myometrial invasion, while the $\mathrm{CC}$ diameter had the greatest $\mathrm{AUC}$ for lymph node metastases $(0.854)$.

\section{DISCUSSION}

To our knowledge, this is the first cohort study to demonstrate that tumor size (in particular, the AP tumor diameter) is a robust indicator of prognosis in patients with EC. ROC curve analysis revealed that the optimal thresholds for predicting prognosis were AP diameter $>28$ $\mathrm{mm}, \mathrm{CC}$ diameter $>52 \mathrm{~mm}$, and TV diameter $>37 \mathrm{~mm}$. Furthermore, we showed that tumor size is significantly associated with deep myometrial invasion (especially AP diameter, threshold value: $28 \mathrm{~mm}$ ), and lymph node metastases (especially CC diameter, threshold value: 52 $\mathrm{mm}$ ). While the prognosis of EC is typically favorable [24], it is poor in some patients. The association between tumor size and prognosis and other clinical prognostic indicators has garnered increasing attention recently. In previous studies, the probability of myometrial invasion increases with the tumor size $[14,15]$. There are two reasons for this. First, when the tumor is growing, it invades the myometrium because there is limited space in the uterine cavity. Second, when the tumor invades the myometrium, the cancer cells first invade the lymphatic or vascular system and next invade the lymph nodes. Previous studies have demonstrated that deep myometrial invasion correlates with surgical factors such as lymph node metastases and distant metastases in EC [25]. However, accurate assessment of myometrial invasion is difficult to obtain using only MRI. For example, it is not uncommon to find that cancers that are classified as stage IA preoperatively turn out to be stage IB postoperatively. If the preoperative diagnosis is uncertain, then the surgical method may be inadequate. For example, whereas pelvic lymphadenectomy is appropriate for stage IB cases (deep myometrial invasion), this should be omitted in patients with a preoperative diagnosis of stage IA and G1. Therefore, it is essential to identify novel factors that are associated with deep myometrial invasion and can be evaluated preoperatively. Tumor size is a potentially useful marker for this purpose that can be obtained with relatively little effort and low interobserver variability.

The same is true of lymph node metastasis. Enlarged lymph nodes seen on preoperative MRI are not always found to be the result of metastasis postoperatively. Several surgical or pathological risk factors for predicting deep myometrial invasion or lymph node metastases have
A AP diameter and PFS (Cut-off value: $28 \mathrm{~mm}$ )

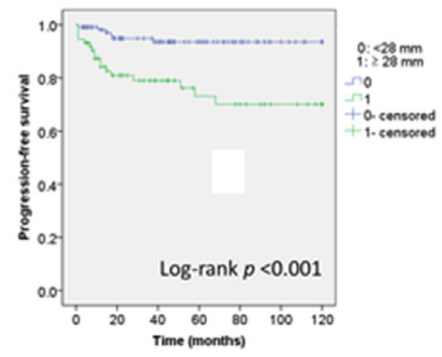

D CC diameter and OS (Cut-off value: $52 \mathrm{~mm}$ )

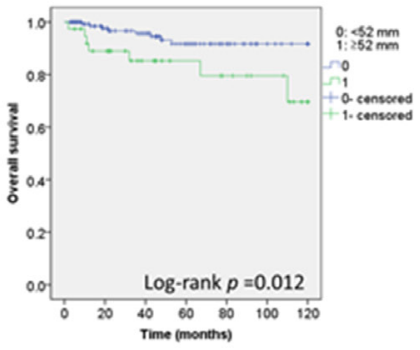

B AP diameter and OS (Cut-off value: $28 \mathrm{~mm}$ )

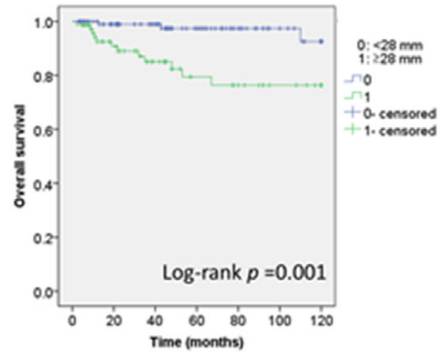

E TV diameter and PFS (Cut-off value: $37 \mathrm{~mm}$ )

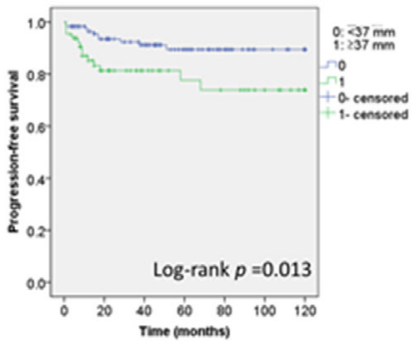

C CC diameter and PFS (Cut-off value: $52 \mathrm{~mm}$ )

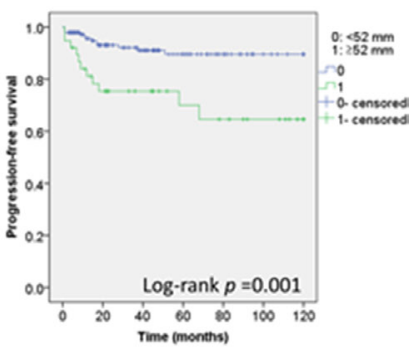

F TV diameter and OS (Cut-off value: $37 \mathrm{~mm}$ )

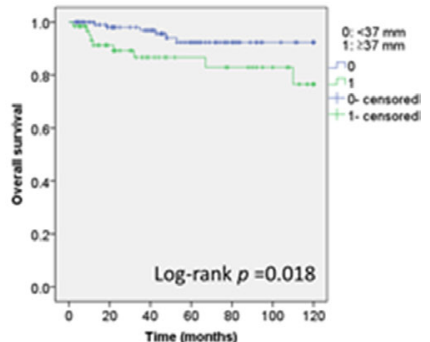

Figure 1: Association of the three tumor size measurements and prognosis. Kaplan-Meier estimates of the usefulness of the AP diameter in the prognosis of PFS (A) and OS (B), the CC diameter in the prognosis of PFS (C) and OS (D), and the TV diameter in the prognosis of PFS (E) and OS (F) in patients with endometrial carcinoma. 
Table 2: Univariate and multivariate analyses of prognostic factors for progression-free survival

\begin{tabular}{lcccccc}
\hline Factor & \multicolumn{3}{c}{ Univariate Analysis } & \multicolumn{3}{c}{ Multivariate Analysis } \\
\cline { 2 - 7 } & $\begin{array}{c}\text { Hazard } \\
\text { Ratio }\end{array}$ & $\mathbf{9 5 \%}$ CI & $\boldsymbol{P}$ & $\begin{array}{c}\text { Hazard } \\
\text { Ratio }\end{array}$ & $\mathbf{9 5 \%}$ CI & $\boldsymbol{P}$ \\
\hline Age at diagnosis, y & 2.379 & $0.978-5.784$ & 0.056 & - & - & - \\
FIGO stage & 9.705 & $3.795-24.816$ & $<0.001$ & 4.441 & $1.463-13.476$ & 0.008 \\
Histology & 3.203 & $1.256-8.171$ & 0.015 & - & - & - \\
Grade & 8.951 & $2.098-38.177$ & 0.003 & 3.193 & $0.680-14.980$ & 0.141 \\
Myometrial invasion & 2.957 & $1.264-6.921$ & 0.012 & - & - & - \\
Lymph metastasis & 10.197 & $3.921-26.518$ & $<0.001$ & - & - & - \\
Venous invasion & 3.726 & $1.589-8.734$ & 0.002 & - & - & - \\
Lymphatic invasion & 7.124 & $2.405-21.106$ & $<0.001$ & 2.770 & $0.795-9.649$ & 0.110 \\
AP diameter & 4.737 & $1.867-12.020$ & 0.001 & - & - & - \\
CC diameter & 3.702 & $1.633-8.392$ & 0.002 & - & - & - \\
TV diameter & 2.729 & $1.195-6.230$ & 0.017 & - & - & - \\
\hline
\end{tabular}

AP, anteroposterior; TV, transverse; CC, craniocaudal; CI, confidence interval; FIGO, International Federation of Gynecology and Obstetrics; N/A, not available.

been reported for EC including histologic type, grade, and tumor extent [26-29]; one model used tumor size greater than $2 \mathrm{~cm}$ according to gross examination of hysterectomy tissue as a predictive factor [26]. Limitations of these models include reliance on surgical and pathological outcomes that cannot be obtained preoperatively. However, direct comparison of macroscopic tumor diameter measurements between fresh tissue and preoperative MRI is difficult because of differences in the planes of the section that can be obtained for tumor measurements and possible distortion of tumor tissue in vivo compared to ex vivo. As a result, the optimal tumor size threshold values may not be transferable from in vivo MRI-based to ex vivo gross section-based measurements. Despite this, both in vivo and ex vivo studies consistently

Correlation between each parameters and deep myometrial invasion A

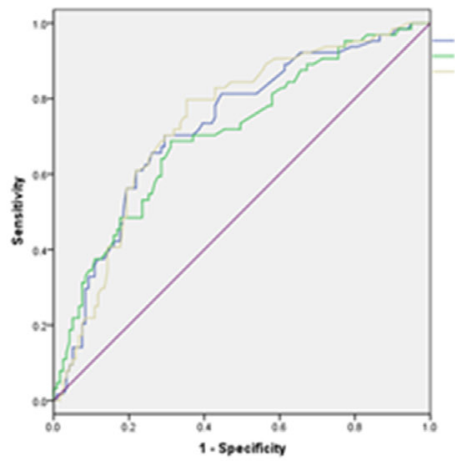

provide evidence of the metastatic potential and negative prognostic impact of large tumor size in EC [26]. Sigmund et al. [29] demonstrated that AP tumor diameter $>2 \mathrm{~cm}$ and $\mathrm{CC}$ tumor diameter $>4 \mathrm{~cm}$ as measured using MRI were associated with prognosis; these results are similar to the findings of the present study [29].

The significance of tumor size as an indicator of pelvic lymphadenectomy in patients with low-risk EC should also be considered. At present, unlike its diagnostic significance, the therapeutic significance of pelvic lymphadenectomy in low-risk EC remains unclear. Previous reports have demonstrated that pelvic lymphadenectomy may be associated with good prognosis [30-34]; however, these studies have not all been randomized controlled studies. Recently, two

Correlation between each parameters and lymph node metastases B

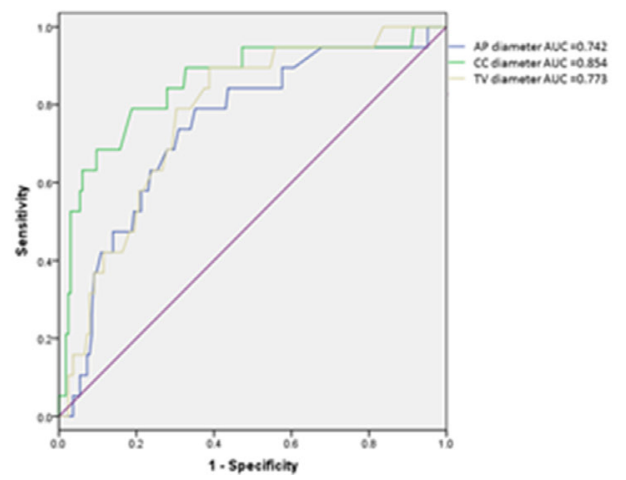

Figure 2: Characteristics for identifying deep endometrial invasion and lymph node metastases. Receiver operating characteristic curves for the three tumor size measurements for identifying deep myometrial invasion (A) and lymph nodemetastases (B) in patients with endometrial carcinoma. 
Table 3: Univariate and multivariate analyses of overall prognostic factors

\begin{tabular}{lcccccc}
\hline Factor & \multicolumn{3}{c}{ Univariate Analysis } & \multicolumn{3}{c}{ Multivariate Analysis } \\
\cline { 2 - 6 } & Hazard Ratio & $\mathbf{9 5 \%}$ CI & $\boldsymbol{P}$ & Hazard Ratio & $\mathbf{9 5 \%}$ CI & $\boldsymbol{P}$ \\
\hline Age at diagnosis, y & 1.548 & $0.551-4.352$ & 0.407 & - & - & - \\
FIGO stage & 9.642 & $3.067-30.311$ & $<0.001$ & 5.756 & $1.708-19.397$ & 0.005 \\
Histology & 3.699 & $1.164-11.755$ & 0.027 & - & - & - \\
Grade & 5.049 & $1.138-22.407$ & 0.033 & - & - & - \\
Myometrial invasion & 2.650 & $0.918-7.644$ & 0.071 & - & - & - \\
Lymph metastasis & 2.529 & $1.474-4.339$ & 0.001 & - & - & - \\
Venous invasion & 3.658 & $1.264-10.589$ & 0.017 & - & - & - \\
Lymphatic invasion & 6.405 & $1.774-23.120$ & 0.005 & - & - & - \\
AP diameter & 6.489 & $1.830-23.013$ & $<0.001$ & 5.285 & $1.110-25.170$ & 0.037 \\
CC diameter & 3.388 & $1.228-9.347$ & 0.012 & - & - & - \\
TV diameter & 3.256 & $1.157-9.165$ & 0.018 & - & - & - \\
\hline AP, and
\end{tabular}

AP, anteroposterior; TV, transverse; CC, craniocaudal; CI, confidence interval; FIGO, International Federation of Gynecology and Obstetrics; N/A, not available.

randomized controlled studies demonstrated that pelvic lymphadenectomy was not associated with prognosis in patients with low-risk EC $[35,36]$. However, in most cases, excluding clearly low-risk cases, lymphadenectomy is considered. Our results indicate that omitting lymphadenectomy in patients with presumed stage I disease with long AP or CC tumor diameter is associated with a risk of recurrence and is therefore not clinically indicated. The results of the present study may be helpful for guiding clinical decision-making regarding lymphadenectomy.

We previously reported that loss of MMR protein expression was identified in 42 of $149(28.2 \%)$ patients with endometrial cancer, and microsatellite instability is a biomarker for immune checkpoint inhibitors in endometrioid endometrial cancer [37]. In this study, we analyzed the relationship between tumor size and prognosis in patients with MMR deficiency $(N=42)$. However, there were no significant differences among them (data not shown). MMR deficient endometrioid carcinoma may behave in a different way in terms of tumor size, but we think that the small sample size may affect this result. Therefore, we must investigate the tumor size significance in a large population of patients with MMR deficiency in the future. Furthermore, other preoperative biomarkers such as p53, hormone receptor, and DNA ploidy status in preoperative biopsies should also be evaluated in the future.

This study has some limitations. First, this was a retrospective study, and therefore, we could not control for all sources of bias. Second, interobserver variability was not evaluated in this study. Third, the tumor growth pattern was not considered in this study. There are various tumor growth patterns such as a surface spreading pattern, an endomyometrium invading pattern, and a polypoid pattern. In previous studies, the influence of the tumor growth pattern on the prognosis was not discussed. We should investigate the influence of tumor growth patterns on the prognosis of EC in the future.

Our findings indicate that tumor size parameters, especially AP diameter, evaluated preoperatively using MRI, are predictive of the presence of deep myometrial invasion and lymph node metastases.

In conclusion, we identified increased AP diameter as a predictive factor of surgical outcomes in patients with EC. Furthermore, tumor size measured preoperatively using MRI is predictive of deep myometrial invasion and lymph node metastases. Based on our findings, preoperative tumor measurements made using MRI may constitute clinically useful markers to assess recurrence risk and guide tailored surgical treatment in EC.

\section{MATERIALS AND METHODS}

\section{Subjects}

This study was approved by the Ethics Committee of Shimane Medical University. We investigated 184 patients with EC who underwent surgery at the University Hospital of Shimane between 1997 and 2016. Patients with insufficient data, nonsurgical treatment, secondary malignancies, hematologic diseases, and those who lacked MRI data were excluded.

Diagnoses were made according to conventional morphological examinations of hematoxylin and eosinstained sections, and tumors were classified according 
to the World Health Organization classification system. The FIGO classification system was used for tumor staging and grading. All patients underwent surgery (total abdominal hysterectomy and bilateral salpingooophorectomy), and those with cancers greater than stage $1 \mathrm{a}$ and grade 1 underwent pelvic lymph node dissection and were administered adjuvant platinum and taxane chemotherapy. Patients with stage $1 \mathrm{a}$ and grade 1 EC did not undergo pelvic lymph node dissection or receive adjuvant chemotherapy.

\section{Measurement of tumor size}

Preoperative MRI was performed 60 days before surgery in all cases. All MRI images were reviewed by two independent radiologists who were blinded to patient data and who conducted assessments for tumor size and histological diagnosis.

The radiologists recorded their assessments of MRI images on a standardized form. Tumor size measurements were made along 3 orthogonal planes: AP and TV diameters were measured using axial contrast-enhanced T1-weighted oblique images (at right angles to the long axis of the uterus), and the $\mathrm{CC}$ diameter was determined using sagittal T2-weighted images (Figure 3A, 3B).

\section{Selection of threshold value}

ROC curve analysis was conducted to establish the threshold value for all tumor size measurements required for diagnosing deep myometrial invasion and lymph node metastases. The sensitivity and specificity of each outcome were plotted to generate ROC curves. The threshold value was selected as the value that was closest to the point with both maximum sensitivity and specificity.

\section{Statistical analysis}

Statistical analyses were carried out using SPSS software (version 19.0 for Windows; IBM Corp., Armonk, NY, USA). ROC curve analysis was applied to determine the threshold value for each tumor size measurement. We used binomial logistic regression analysis for univariate analysis for ordered categorical variables. We used the following clinical factors for modeling: patient age at diagnosis ( $<60$ vs. $\geq 60$ years), stage (I/II vs. III/IV), histologic type (endometrioid vs. others), tumor grade ( 1 vs. $2 / 3$ ), myometrial invasion ( $<1 / 2$ vs. $\geq 1 / 2$ ), lymph metastasis, venous invasion, lymphatic invasion, and three tumor size measurements (AP, TV, and CC diameter).

ROC analysis was used to determine the usefulness of the three tumor size measurements for the diagnosis of deep myometrial invasion and lymph node metastases. The optimal threshold values (rounded to the nearest centimeter) were chosen as those producing the best separation of the Youden index between the groups.

PFS and OS were the endpoints of the analysis. PFS indicates the time between initial diagnosis and initial recurrence of the disease. Patients with no recurrence at their most recent follow-up were censored at the followup. OS indicates the time between initial diagnosis and death. Patients who were living at their most recent follow-up were censored at the follow-up. Kaplan-Meier curves and log-rank tests were used to plot the survival data and determine the statistical significance of survival differences. We entered variables that were significant $(P<0.05)$ in the univariate analysis into the multivariate analysis. Cox's proportional hazards model was used for prognostic analysis. Data of patients who were lost to follow-up were censored. All reported $P$ values were twosided, and a $P$ value $<0.05$ was considered significant.
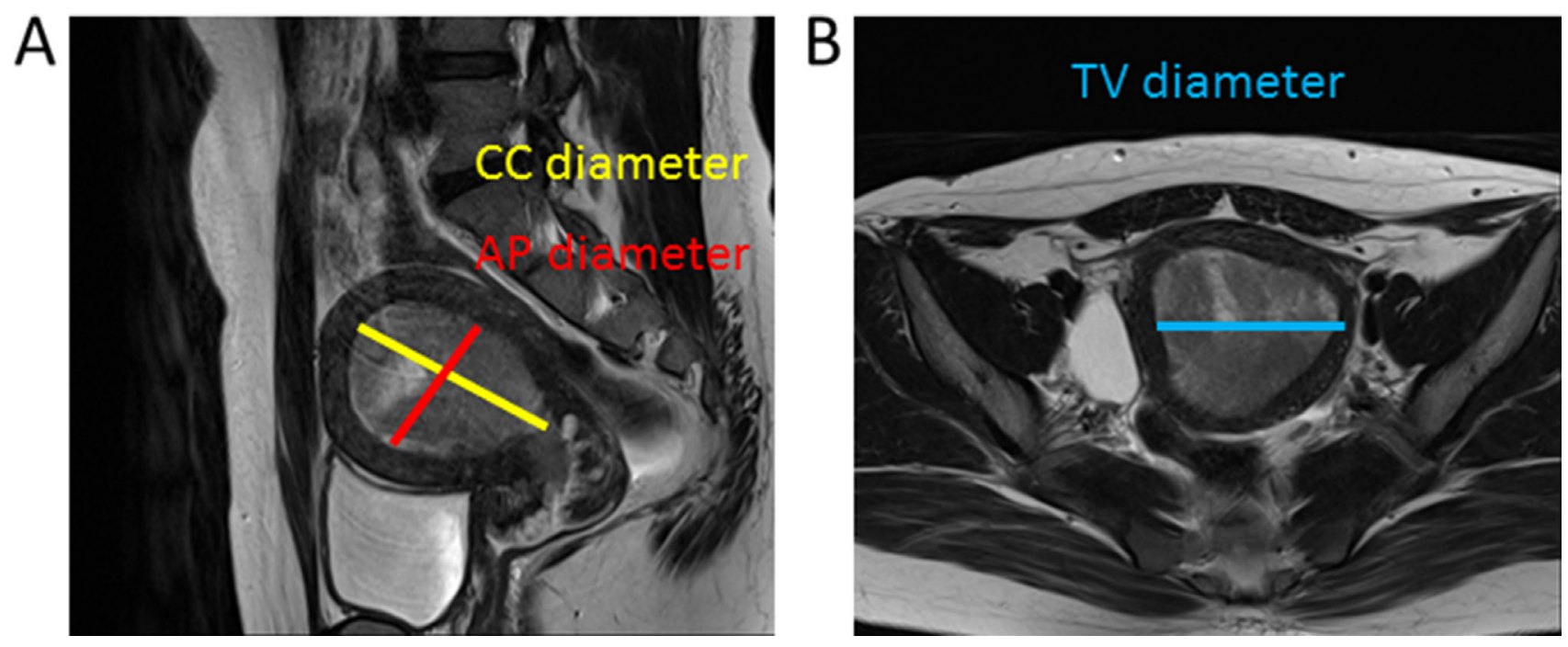

Figure 3: Images used to determine tumor diameter. The maximum tumor diameters were determined along three orthogonal planes. AP and maximum CC diameter were determined using the sagittal oblique T2-weighted image (A), and the TV diameter was determined using the coronal image $(\mathbf{B})$. 


\section{Abbreviations}

AP, anteroposterior; AUC, area under the curve; $\mathrm{CC}$, craniocaudal; EC, endometrial carcinoma; FIGO, The International Federation of Gynecology and Obstetrics; MRI, magnetic resonance imaging; OS, overall survival; PFS, progression-free survival; ROC, receiver operating characteristic; TV, transverse.

\section{CONFLICTS OF INTEREST}

The authors declare no conflicts of interest.

\section{REFERENCES}

1. Ferlay J, Soerjomataram I, Dikshit R, Eser S, Mathers C, Rebelo M, Parkin DM, Forman D, Bray F. Cancer incidence and mortality worldwide: sources, methods, and major patterns in GLOBOCAN 2012. Int J Cancer. 2015; 136:E359-E386.

2. Burke WM, Orr J, Leitao M, Salom E, Gehrig P, Olawaiye AB, Brewer M, Boruta D, Villella J, Herzog T, Abu Shahin F; SGO Clinical Practice Endometrial Cancer Working Group; Society of Gynecologic Oncology Clinical Practice Committee. Endometrial cancer: A review and current management strategies: Part I. Gynecol Oncol. 2014; 134:385-392.

3. Ho CM, Chien TY, Huang SH, Wu CJ, Shih BY, Chang SC. Multivariate analysis of the prognostic factors and outcomes in early cervical cancer patients undergoing radical hysterectomy. Gynecol Oncol. 2004; 93:458-464.

4. Kamura T, Tsukamoto N, Tsuruchi N, Saito T, Matsuyama T, Akazawa K, Nakano H. Multivariate analysis of the histopathologic prognostic factors of cervical cancer in patients undergoing radical hysterectomy. Cancer. 1992; 69:181-186.

5. Takeda N, Sakuragi N, Takeda M, Okamoto K, Kuwabara M, Negishi H, Okiawa M, Yamamoto R, Yamada H, Fujimoto S. Multivariate analysis of histopathologic prognostic factors for invasive cervical cancer treated with radical hysterectomy and systematic retroperitoneal lymphadenectomy. Acta Obstet Gynecol Scand. 2002; 81:1144-1151.

6. Fotiou S, Vlahos $\mathrm{N}$, Kondi-Pafiti A, Zarganis $\mathrm{P}$, Papakonstantinou K, Creatsas G. Intraoperative gross assessment of myometrial invasion and cervical involvement in endometrial cancer: Role of tumor grade and size. Gynecol Oncol. 2009; 112:517-520.

7. Lo KW, Cheung TH, Yim SF, Chung TK. Preoperative hysteroscopic assessment of cervical invasion by endometrial carcinoma: a retrospective study. Gynecol Oncol. 2001; 82:279-282.

8. Haldorsen IS, Berg A, Werner HM, Magnussen IJ, Helland H, Salvesen OO, Trovik J, Salvesen HB. Magnetic resonance imaging performs better than endocervical curettage for preoperative prediction of cervical stromal invasion in endometrial carcinomas. Gynecol Oncol. 2012; 126:413-418.

9. Burghardt E, Pickel H. Local spread and lymph node involvement in cervical cancer. Obstet Gynecol. 1978; 52:138-145.

10. Elledge RM, McGuire WL, Osborne CK. Prognostic factors in breast cancer. Semin Oncol. 1992; 19:244-253.

11. Scoll BJ, Wong YN, Egleston BL, Kunkle DA, Saad IR, Uzzo RG. Age, tumor size and relative survival of patients with localized renal cell carcinoma: a surveillance, epidemiology and end results analysis. J Urol. 2009; 181:506-511.

12. Nguyen MM, Gill IS. Effect of renal cancer size on the prevalence of metastasis at diagnosis and mortality. J Urol. 2009; 181:1020-1027.

13. Gusberg SB, Jones HC Jr, Tovell HM. Selection of treatment for corpus cancer. Am J Obstet Gynecol. 1960; 80:374-380.

14. Schink JC, Rademaker AW, Miller DS, Lurain JR. Tumor size in endometrial cancer. Cancer. 1991; 67:2791-2794.

15. Mariani A, Webb MJ, Keeney GL, Haddock MG, Calori G, Podratz KC. Low-risk corpus cancer: is lymphadenectomy or radiotherapy necessary? Am J Obstet Gynecol. 2000; 182:1506-1519.

16. Schink JC, Lurain JR, Wallemark CB, Chmiel JS. Tumor size in endometrial cancer: a prognostic factor for lymph node metastasis. Obstet Gynecol. 1987; 70:216-219.

17. Mariani A, Dowdy SC, Cliby WA, Gostout BS, Jones MB, Wilson TO, Podratz KC. Prospective assessment of lymphatic dissemination in endometrial cancer: a paradigm shift in surgical staging. Gynecol Oncol. 2008; 109:11-18.

18. Lurain JR, Rice BL, Rademaker AW, Poggensee LE, Schink JC, Miller DS. Prognostic factors associated with recurrence in clinical stage I adenocarcinoma of the endometrium. Obstet Gynecol. 1991; 78:63-69.

19. Shah C, Johnson EB, Everett E, Tamimi H, Greer B, Swisher E, Goff B. Does size matter? Tumor size and morphology as predictors of nodal status and recurrence in endometrial cancer. Gynecol Oncol. 2005; 99:564-570.

20. Creasman W. Revised FIGO staging for carcinoma of the endometrium. Int J Gynaecol Obstet. 2009; 105:109.

21. Soliman PT, Frumovitz M, Spannuth W, Greer MJ, Sharma S, Schmeler KM, Ramirez PT, Levenback CF, Ramondetta LM. Lymphadenectomy during endometrial cancer staging: practice patterns among gynecologic oncologists. Gynecol Oncol. 2010; 119:291-294.

22. Kumar S, Bandyopadhyay S, Semaan A, Shah JP, Mahdi H, Morris R, Munkarah A, Ali-Fehmi R. The role of frozen section in surgical staging of low risk endometrial cancer. PLoS One. 2011; 6:e21912.

23. Frumovitz M, Slomovitz BM, Singh DK, Broaddus RR, Abrams J, Sun CC, Bevers M, Bodurka DC. Frozen section analyses as predictors of lymphatic spread in patients with early-stage uterine cancer. J Am Coll Surg. 2004; 199:388-393. 
24. Siegel R, Naishadham D, Jemal A. Cancer statistics, 2013. CA Cancer J Clin. 2013; 63:11-30.

25. Salvesen HB, Haldorsen IS, Trovik J. Markers for individualised therapy in endometrial carcinoma. Lancet Oncol. 2012; 13:e353-e361.

26. Mariani A, Webb MJ, Keeney GL, Lesnick TG, Podratz KC. Surgical stage I endometrial cancer: predictors of distant failure and death. Gynecol Oncol. 2002; 87:274-280.

27. Creasman WT, Morrow CP, Bundy BN, Homesley HD, Graham JE, Heller PB. Surgical pathologic spread patterns of endometrial cancer. A Gynecologic Oncology Group Study. Cancer. 1987; 60:2035-2041.

28. Kang S, Lee JM, Lee JK, Kim JW, Cho CH, Kim SM, Park SY, Park CY, Kim KT. How low is low enough? Evaluation of various risk-assessment models for lymph node metastasis in endometrial cancer: a Korean multicenter study. J Gynecol Oncol. 2012; 23:251-256.

29. Ytre-Hauge S, Husby JA, Magnussen IJ, Werner HM, Salvesen OO, Bjorge L, Stefansson IM, Salvesen HB, Haldorsen IS. Preoperative tumor size at MRI predicts deep myometrial invasion, lymph node metastases, and patient outcome in endometrial carcinomas. Int J Gynecol Cancer. $2015 ; 25: 459-466$

30. Kilgore LC, Partridge EE, Alvarez RD, Austin JM, Shingleton HM, Noojin F 3rd, Conner W. Adenocarcinoma of the endometrium: survival comparisons of patients with and without pelvic node sampling. Gynecol Oncol. 1995; 56:29-33.

31. Fanning J. Long-term survival of intermediate risk endometrial cancer (stage IG3, IC, II) treated with full lymphadenectomy and brachytherapy without teletherapy. Gynecol Oncol. 2001; 82:371-374.
32. Takeshima N, Hirai Y, Tanaka N, Yamawaki T, Yamauchi K, Hasumi K. Pelvic lymph node metastasis in endometrial cancer with no myometrial invasion. Obstet Gynecol. 1996; 88:280-282.

33. Larson DM, Broste SK, Krawisz BR. Surgery without radiotherapy for primary treatment of endometrial cancer. Obstet Gynecol. 1998; 91:355-359.

34. Cragun JM, Havrilesky LJ, Calingaert B, Synan I, Secord AA, Soper JT, Clark-Pearson DL, Berchuck A. Retrospective analysis of selective lymphadenectomy in apparent early-stage endometrial cancer. J Clin Oncol. 2005; 23:3668-36675.

35. Kitchener H, Swart AM, Qian Q, Amos C, Parmar MK; ASTEC study group. Efficacy of systematic pelvic lymphadenectomy in endometrial cancer (MRC ASTEC trial): a randomised study. Lancet. 2009; 373:125-136.

36. Benedetti Panici P, Basile S, Maneschi F, Alberto Lissoni A, Signorelli M, Scambia G, Angioli R, Tateo S, Mangili G, Katsaros D, Garozzo G, Campagnutta E, Donadello $\mathrm{N}$, et al. Systematic pelvic lymphadenectomy vs. no lymphadenectomy in early-stage endometrial carcinoma: randomized clinical trial. J Natl Cancer Inst. 2008; 100:1707-1716.

37. Yamashita H, Nakayama K, Ishikawa M, Nakamura K, Ishibashi T, Sanuki K, Ono R, Sasamori H, Minamoto T, Iida K, Sultana R, Ishikawa N, Kyo S. Microsatellite instability is a biomarker for immune checkpoint inhibitors in endometrial cancer. Oncotarget. 2018; 9:5652-5664. https://doi.org/10.18632/oncotarget.23790. 